\title{
Bearing performance degradation assessment based on the contact stress and deformation
}

\author{
Hu Qingzhong ${ }^{1,2, a}$, Chu Fulei ${ }^{2, b}$ \\ ${ }^{1}$ Department of Mechanical Engineering, Tsinghua University ,Beijing 100084 ,P. R. China. \\ ${ }^{2}$ Key laboratory for the space launching site reliability technology; Xichang; 615000, P. R. China. \\ ahuqingz@163.combhofl@mail.tsinghua.edu.cn
}

Keywords: Performance degradation assessment. Contact stress. Deformation.

Abstract. Aimed at the same bearing in the same condition will appear different failure modes for single bearing performance degradation evaluation, this paper puts forward a new performance degradation assessment method based on contact stress and deformation, which based on the Hertz's point contact theory and line contact theory, through calculation, the calculated with roller bearings inside the circle contact area size, produced contact stress and contact deformation by monitoring, to judge the parameter value differences with the theoretical performance degradation to achieve bearing assessment, and to $7208 \mathrm{c}$ bearing, for example are assessment.

\section{Introduction}

Rolling bearing is one of the most modern industrial applications key components, supporting the rotating member, plays a role in load and transfer the load to bear, once failure occurs, it will affect the state and the safe operation of the entire system, resulting in a series of sabotage. Although the use of a sufficiently long bearing design life, but any bearings having a zero probability of unlimited durability; same batch the same rolling bearing, operating under the same load, same speed, same lubrication and the same environmental conditions, will It appears in different ways at different times of failure [1]. However, before bearing failure occurs, usually through a series of different stages of degradation of performance, if they can at this stage be able to effectively monitor the performance degradation degree bearing, we can take the appropriate measures to prevent and avoid the occurrence of failure, so work system to maximize the effectiveness [2] [3].

Since the elastic contact theory [4] proposed, this theory have been able to accurately solve the Rolling stress and deformation of each element in the work process. With the rapid development in recent years, intelligent sensor technology with modern sensor Rolling internal stress and deformation of the contact area detecting possible [5]. So theoretically the working status of the rolling bearing analysis, from the reality of the state monitoring, explores the rolling bearing performance degradation characteristics it has great significance.

\section{Rolling bearing contact area of stress and deformation}

Rolling bearing rely on contact with the rolling elements and the inner and outer ring to bear the load, since the contact area of the rolling element and inner and outer rings is very small, so this will have a huge contact area and stress certain deformation. If you can calculate and measure the magnitude of stress and deformation of the contact area can be effectively degraded performance bearings for effective evaluation.

\section{Introduction to the theory of Hertz contact}

Hertz in the calculation of the first point of contact stress and deformation made the following assumptions: the material is homogeneous; be deformed within the elastic limit; the contact force perpendicular to the plane; the size of the contact area is much smaller than the size of the object. Then the comprehensive application of the theory of infinitesimal elasticity cubes establish static equilibrium equation, deformation equation, shear strain equations, etc., and the introduction of a series of auxiliary equation, and finally get the normal stress equation and a consistent set of equations. Using 
these equations, known under the stress-strain state of stress can be solved and the internal elastic inside the object.

Hertz finally assume the shape of the deformed surface is a rotating ellipsoid (Fig. 1), by positive stress equation is solved, finally obtained compressive stress ( $\sigma$ )of the contact area, the contact area of the ellipse parameters long axle(a), axle and short(b) amount of elastic deformation $(\sigma)$ for:

$$
\begin{array}{ccc}
\sigma=\frac{3 Q}{2 \pi a b}\left[1-\left(\frac{x}{a}\right)^{2}-\left(\frac{y}{b}\right)^{2}\right]^{\frac{1}{2}} & \text { (1) } \quad a=a^{*}\left[\frac{3 Q}{2 \sum \rho}\left(\frac{\left(1-\mu_{\mathrm{I}}^{2}\right)}{E_{\mathrm{I}}}+\frac{\left(1-\mu_{\mathrm{II}}^{2}\right)}{E_{\mathrm{II}}}\right)\right]^{\frac{1}{3}} \\
b=b^{*}\left[\frac{3 Q}{2 \sum \rho}\left(\frac{\left(1-\mu_{\mathrm{I}}^{2}\right)}{E_{\mathrm{I}}}+\frac{\left(1-\mu_{\mathrm{II}}^{2}\right)}{E_{\mathrm{II}}}\right)\right]^{\frac{1}{3}} & \text { (3) } \quad \delta=\delta^{*}\left[\frac{3 Q}{2 \sum \rho}\left(\frac{\left(1-\mu_{\mathrm{I}}^{2}\right)}{E_{\mathrm{I}}}+\frac{\left(1-\mu_{\mathrm{II}}^{2}\right)}{E_{\mathrm{II}}}\right)\right]^{\frac{2}{3}} \frac{\sum \rho}{2}
\end{array}
$$

Where: $\mu$ is Poisson's ratio, $E$ is elastic modulus, $Q$ is the law to force, $\sum \rho$ is curvature sum , $a^{*}, b^{*}$ and $\delta^{*}$ are normalized parameters, including:

$$
\begin{array}{ccc}
\sum \rho=\rho_{I 1}+\rho_{I 2}+\rho_{I I 1}+\rho_{I I 2} & \text { (5) } & a^{*}=\left(\frac{2 \kappa^{2} \mathrm{E}}{\pi}\right)^{\frac{1}{3}} \\
b^{*}=\left(\frac{2 \mathrm{E}}{\pi \kappa}\right)^{\frac{1}{3}} & \text { (7) } & \delta^{*}=\frac{2 \mathrm{~F}}{\pi}\left(\frac{\pi}{2 \kappa^{2} \mathrm{E}}\right)^{\frac{1}{3}}
\end{array}
$$

In the formula, $\kappa$ elliptical eccentricity, $\mathrm{E}$ and $\mathrm{F}$ are the first class and second class complete elliptic integral, curvature function is introduced in contact form $F(\rho)$ :

$$
F(\rho)=\frac{\left(\rho_{I 1}-\rho_{I 2}\right)+\left(\rho_{I I 1}-\rho_{I I 2}\right)}{\sum \rho}
$$

It is also the ellipse parameters $a$ and $b$ functions:

$$
F(\rho)=\frac{\left(\kappa^{2}+1\right) \mathrm{E}-2 \mathrm{~F}}{\left(\kappa^{2}-1\right) \mathrm{E}}
$$

Given eccentricity ellipse parameters $\kappa$, we can calculate the value of $F(\rho)$ corresponding.

This can be established $a^{*}, b^{*}, \delta^{*}$ and $F(\rho)$ correspondence. Its value can be directly fig 2 , may also be related tables $[1,6]$ directly or obtained by interpolation.

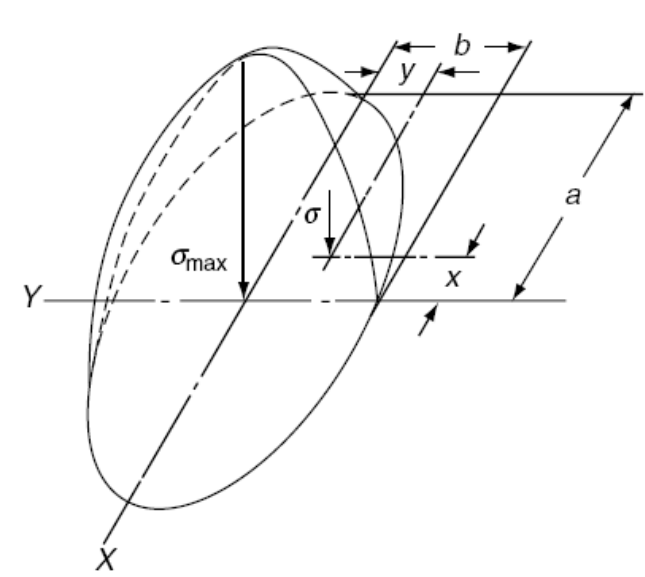

Fig1 point pressure stress distribution on contact ellipse

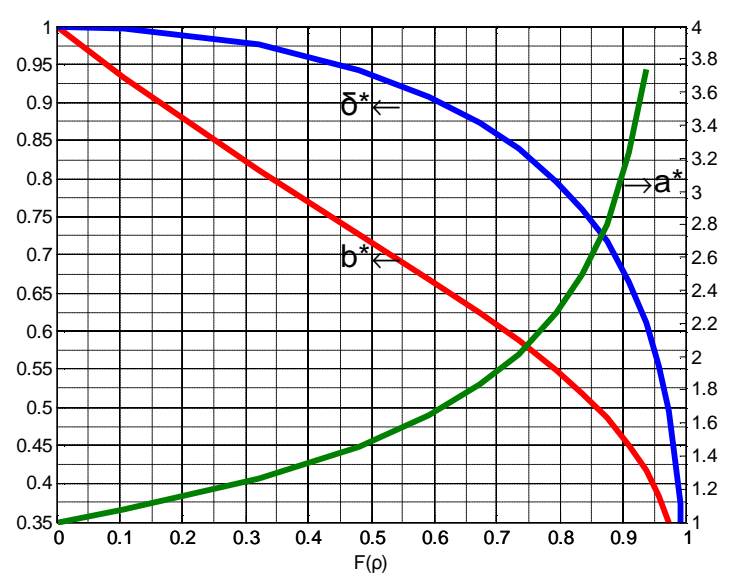

Fig $2 \mathrm{a} *, \mathrm{~b} *, \delta *$ with $\mathrm{F}(\rho)$ diagram

For an ideal line contact, the contact body I length must be equal to the length of the contact body II. In this case, $\kappa$ tends to infinity, the contact stress distribution area into semi-elliptical cylindrical as shown in Fig 3. 
The contact area and the contact normal stress line width:

$$
\sigma=\frac{2 Q}{\pi l b}\left[1-\left(\frac{y}{b}\right)^{2}\right]^{\frac{1}{2}}
$$

$$
b=\left[\frac{4 Q}{\pi l \sum \rho}\left(\frac{\left(1-\mu_{\mathrm{I}}^{2}\right)}{E_{\mathrm{I}}}+\frac{\left(1-\mu_{\mathrm{II}}^{2}\right)}{E_{\mathrm{II}}}\right)\right]^{\frac{1}{2}}
$$

\section{Surface contact area of rolling bearing, the stress and deformation calculation}

Rolling bearing surface contact area, the stress and deformation calculation

For the inside of the rolling bearing contact problem, the Hertz assumptions are satisfied, therefore can be directly applied to rolling bearing.

Internal structure diagram and force Rolling shown in Figure 4.
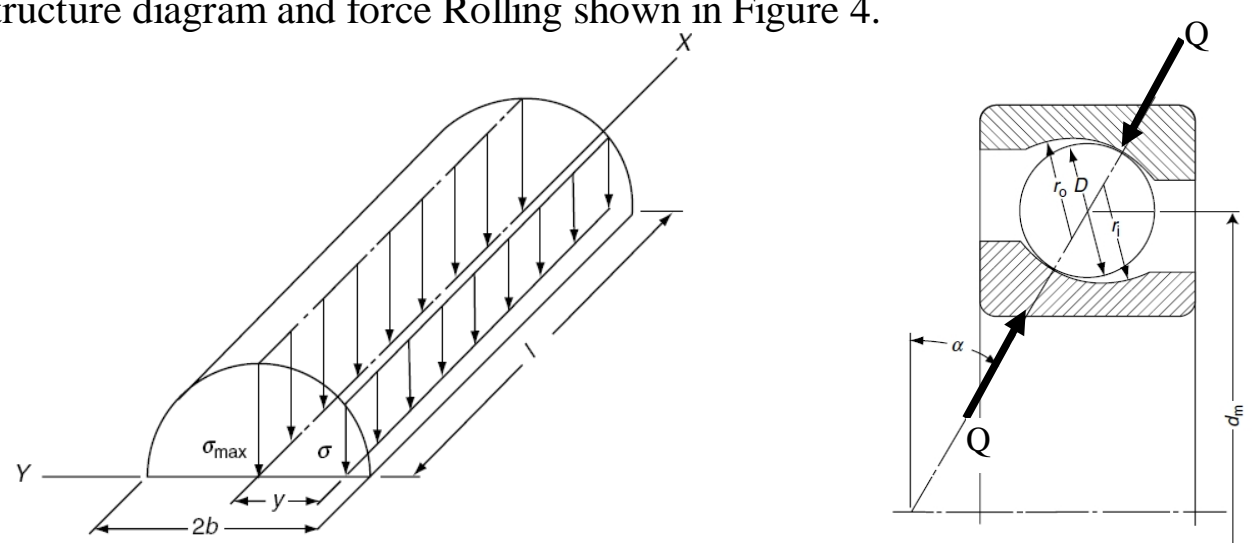

Figu 3 line semi-elliptical surface stress distribution Fig 4 Rolling geometry and force diagrams

Curvature and the curvature difference between the inner ring and the rolling element

For the rolling element radius perpendicular to the plane of the two main

$$
r_{\mathrm{I} 1}=r_{\mathrm{I} 2}=\frac{1}{2} D
$$

Where, $D$ is the rolling element diameter.

For the inner radius corresponding to the main plane of the two rolling element inside is:

The radius of inner ring main plane I for:

$$
r_{\mathrm{III}}=\frac{1}{2} d_{i}=\frac{1}{2}\left(\frac{d_{m}}{\cos \alpha}-D\right)
$$

Where: $d_{i}$ is the rolling bearing pitch diameter ; $d_{m}$ is the rolling bearing pitch diameter; $\alpha$ is the rolling elements and the inner and outer rings of the contact angle.

The radius of the inner main plane II as follows:

$$
r_{\text {II } 2}=r_{i}=f_{i} D
$$

Where $r_{i}$ is the radius of curvature of the inner rolling groove, $f_{i}$ is the auxiliary variables make $f_{i}=r_{i} / D$

$$
\begin{aligned}
& \gamma=\frac{D \cos \alpha}{d_{m}} \\
& \rho_{\mathrm{II} 1}=\frac{2}{D}\left(\frac{\gamma}{1-\gamma}\right)
\end{aligned}
$$

$$
\begin{aligned}
& \rho_{\mathrm{I} 1}=\rho_{\mathrm{I} 2}=\frac{2}{D} \\
& \rho_{\mathrm{II} 2}=-\frac{1}{f_{i} D}
\end{aligned}
$$

Therefore, the Rolling element contact with inner ring raceway's curvature sum $\sum \rho$ and curvature difference $F(\rho)$ :

$$
\sum \rho_{i}=\frac{1}{D}\left(4-\frac{1}{f_{i}}+\frac{2 \gamma}{1-\gamma}\right) \quad(20) \quad F(\rho)_{i}=\frac{\frac{1}{f_{i}}+\frac{2 \gamma}{1-\gamma}}{4-\frac{1}{f_{i}}+\frac{2 \gamma}{1-\gamma}}
$$




\section{the rolling element and the outer ring's curvature sum and curvature difference}

when the rolling element and the outer ring raceway contact, radius of curvature of the rolling elements and the same as above, that is $\rho_{\mathrm{I} 1}=\rho_{\mathrm{I} 2}=\frac{2}{D}$, while the radius of curvature of the outer ring

$$
\rho_{\mathrm{II} 1}=-\frac{2}{D}\left(\frac{\gamma}{1+\gamma}\right)
$$

$$
\rho_{\mathrm{II} 2}=-\frac{1}{f_{o} D}
$$

Therefore, the rolling element and the outer ring's curvature sum and curvature difference

$$
\sum \rho_{o}=\frac{1}{D}\left(4-\frac{1}{f_{o}}-\frac{2 \gamma}{1+\gamma}\right)
$$

$$
F(\rho)_{o}=\frac{\frac{1}{f_{o}}-\frac{2 \gamma}{1+\gamma}}{4-\frac{1}{f_{o}}-\frac{2 \gamma}{1+\gamma}}
$$

\section{Rolling bearing contact area, deformation and stress calculation}

After calculating the inner and the outer ring's curvature sum and curvature difference, Check fig 2 to $a^{*}, b^{*}$ and $\delta^{*}$ values.

As the material for the rolling bearing steel, the elastic modulus of the same two principal planes, are: $E_{1}=E_{2}=207 \times 10^{3} M P a$,Poisson's ratio of: $\mu_{\mathrm{I}}=\mu_{\mathrm{II}}=0.3$, these values into the equation (1) to (4), can get rolling ball bearing contact area and contact deformation is:

$$
\begin{array}{ccc}
a=0.0236 a^{*}\left(\frac{Q}{\sum \rho}\right)^{\frac{1}{3}} & \text { (26) } & b=0.0236 b^{*}\left(\frac{Q}{\sum \rho}\right)^{\frac{1}{3}} \\
\delta=2.79 \times 10^{-4} \delta^{*} Q^{\frac{2}{3}} \sum \rho^{\frac{1}{3}} & \text { (28) } & \sigma=\frac{3 Q}{2 \pi a b}\left[1-\left(\frac{x}{a}\right)^{2}-\left(\frac{y}{b}\right)^{2}\right]^{\frac{1}{2}}
\end{array}
$$

For the rolling bearings, the maximum compressive stress occurs at the geometric center of the rolling element and raceway contact, the size of

$$
\sigma_{\max }=\frac{3 Q}{2 \pi a b}
$$

Internal size of the contact area of the rolling elements by force into the equation (26) to (30) can be calculated ball bearings, contact stresses and contact deformation.

\section{bearing performance degradation monitoring and assessment}

Since the internal rolling bearing contact area, size and deformation are calculated, so as long as the sensor detects the actual value of these parameters can be performed on the bearing performance degradation assessment.

In recent years, the development of materials, thin-film technology and MEMS technology, the design and the micro thin-film sensor directly encapsulated within the bearing as possible [3]. Between the thin film sensor grid, it is easy to put the two contact surfaces, with a small, fast dynamic response, high sensitivity, ease of integration, etc., can be completely adapted to the requirements of intelligent bearing [3], and in turn capable of interference to a minimum. Therefore, the film can be grid sensors embedded between the inner and outer raceways, force monitoring range by a dot on the film on mesh sensor points can know the force area within the bearing raceway, through the lattice points by force and deformation can know the bearing stress and deformation, it is possible to carry out performance monitoring and bearing performance degradation assessment.

1) When the actual measured surface contact range is greater than the theoretical calculation of reach of, and accompanied by the growth trend, but less than the maximum contact range, bearing performance degraded; when exceeding the maximum reach of bearing failure occurs. 
2) When the stress measured surface contact area is greater than the theoretical calculation of the bearing surface of normal stress, accompanied by an increasing trend, bearing performance degraded; when the maximum value of the contact normal stress is greater than the stress measured when bearing bear, bearing failure.

3) When the measured surface contact deformation of the contact area is greater than the calculated bearing surface contact deformation and the elastic deformation can not be fully restored, when accompanied by an increasing trend, bearing performance degraded; when the maximum amount of deformation exceeds the permitted bearing failure occurs.

\section{Evaluation Examples}

$7208 \mathrm{C}$ angular contact ball bearing inner diameter is $40 \mathrm{~mm}$, outer diameter is $80 \mathrm{~mm}$, thickness is $18 \mathrm{~mm}$, contact angle is 120 , the rolling ball diameter is $12.7 \mathrm{~mm}$, the rolling groove outer diameter is $74.42 \mathrm{~mm}$,both the inner and outer groove curvature radius is $6.54 \mathrm{~mm}$, so,

$$
d_{m}=d_{o}-D=60 \mathrm{~mm} \quad \text { (31) } f_{i}=f_{o}=r / D=0.515 \quad \text { (32) } \quad \gamma=D \cos \alpha / d_{m}=0.207
$$

By the formula (20) and is (21), the inner raceway curvature and curvature deviation

$$
\sum \rho_{i}=\frac{1}{D}\left(4-\frac{1}{f_{i}}+\frac{2 \gamma}{1-\gamma}\right)=0.203 \mathrm{~mm}^{-1}(34) \quad F(\rho)_{i}=\frac{\frac{1}{f_{i}}+\frac{2 \gamma}{1-\gamma}}{4-\frac{1}{f_{i}}+\frac{2 \gamma}{1-\gamma}}=0.955
$$

By the formula (24) and (25), with outer raceway curvature and curvature difference

$$
\sum \rho_{o}=\frac{1}{D}\left(4-\frac{1}{f_{o}}-\frac{2 \gamma}{1+\gamma}\right)=0.135 \mathrm{~mm}^{-1} \quad \text { (36) } \quad F(\rho)_{o}=\frac{\frac{1}{f_{o}}-\frac{2 \gamma}{1+\gamma}}{4-\frac{1}{f_{o}}-\frac{2 \gamma}{1+\gamma}}=0.932
$$

According to the above values Chaturvedi 2 can be obtained, and the value

$a_{i}^{*}=4.30, b_{i}^{*}=0.39, \delta_{i}^{*}=0.56 \quad a_{o}^{*}=3.63, b_{o}^{*}=0.42, \delta_{o}^{*}=0.62$

By the formula (26) and (27), the inner ring contact with is

$$
a_{i}=0.1736 Q^{\frac{1}{3}} \mathrm{~mm}
$$

$$
b_{i}=0.0156 Q^{\frac{1}{3}} \mathrm{~mm}
$$

When the rolling element with stand different forces, the contact surface shape shown in Fig 5.

Figure 5 can be assessed: physical shape when the detected contact as shown in the size of the deformation, bearing work; when the contact deformation greater than the real figure, but the figure in the elliptic cylinder (blue dotted line) inside, and when there is growing trend in bearing performance degradation; when the contact deformation beyond the elliptical cylinder and over the height of the ellipse (the yellow plane), bearing failure occurs.

The ellipse parameters $a$ and $b$ values are substituted into equation (29), then under certain circumstances the force, the inner ring and the rolling body contact stress distribution at Figure 6.

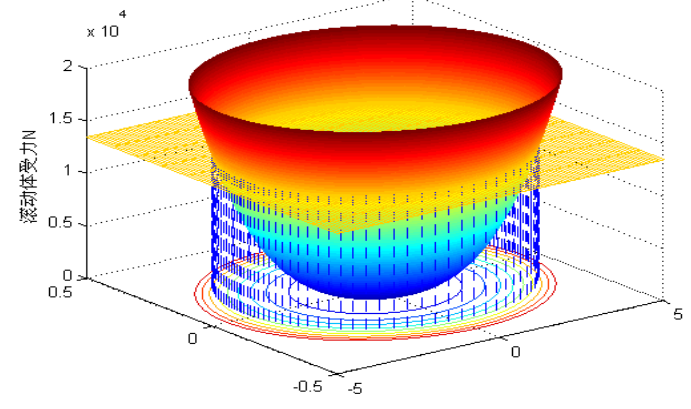

Fig 5 the innerring raceway contact surface shape

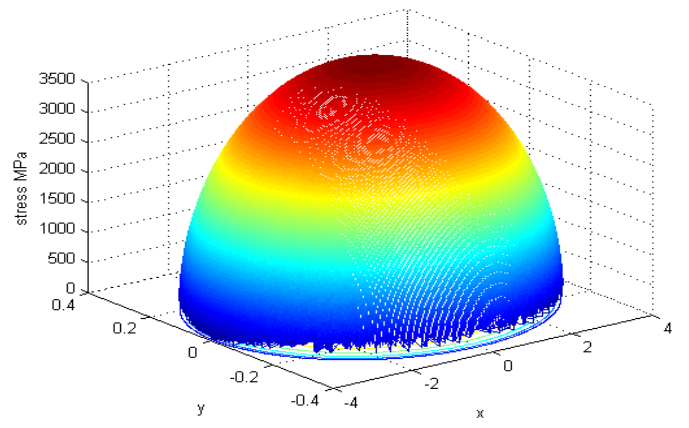

Fig 6 the inner raceway contact stress distribution 
From the figure, the maximum normal stress occurs in the contact center, its size by the formula (30) is obtained:

$$
\sigma_{i \max }=\frac{3 Q}{2 \pi a b}=177.48 Q^{\frac{1}{3}} M P a
$$

Similarly, the elastic deformation of the contact by the formula (28) is obtained:

$$
\delta_{i}=0.000279 \delta^{*} Q^{\frac{2}{3}} \sum \rho^{\frac{1}{3}}=0.000092 Q^{\frac{2}{3}} \mathrm{~mm}
$$

Maximum normal stress and elastic under different loading conditions modification shown in Fig 7.

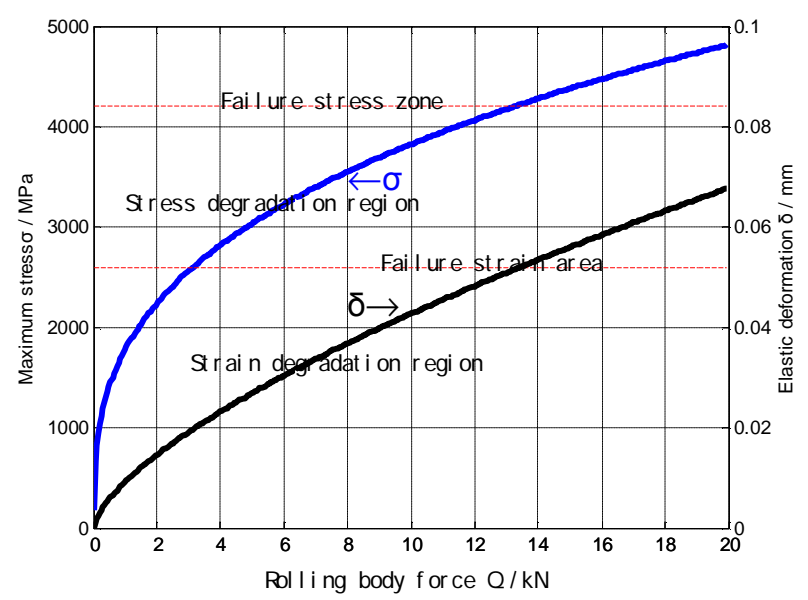

Fig. 7208 bearing raceway contact maximum normal stress and the elastic deformation maps

Fig 7 can be assessed: the force in certain circumstances, when the maximum contact stress and the elastic deformation of the curve shown in FIG bearing normal performance; when the maximum contact stress and elastic deformation curve higher figure, but in failure when under the curve, and tends to increase, in bearing performance degradation; when the maximum contact stress and elastic deformation curve beyond failure, bearing failure occurs.

\section{Conclusion}

This article outlines the Hertz contact theory, the bearing contact stress calculation methods and contact deformation, and points out ways of bearing performance degradation monitoring, indicators and methods proposed bearing performance degradation assessment. The new approach has some theoretical significance and application value of bearing performance degradation assessment, prevention of bearings, damage to the equipment has a positive preventive significance.

\section{references}

[1] Tedric A. Harris ,Michael N. Kotzalas. Rolling Bearing analysis fifth edition: essential concepts of bearing technology[M]. Boca Raton , FL : Taylor \& Francis , c2007

[2] Djurdjanovic D,Lee J,Ni J. Watchdog Agent an infotronics based prognostics approach for product performance degradation assessment and prediction. Advanced Engineering Informatics 2003:17(3-4):109-125.

[3] Guo Lei, Chen Jin. A Rewiew on machinery performance degradatinon assessment and prediction,Journal of Vibration and Shock,2008,27(S):139 142

[4] Hertz.H,On the contact of rigid elastic solids and on hardness, Miscellaneous Papers, MacMillan,London,1896:163- 183.

[5] ZHANG Yichen, LIU Xidong, BA Dechun, GAO Hang. Thin film sensor application in smart bearing[J], VACUUM,2003 , (6):6-10 